\title{
Collagen arrangement in hepatic granuloma in mice infected with Schistosoma mansoni: dependence on fiber radiation centers
}

\author{
H.L. Lenzi ${ }^{1}$, E. Kimmel ${ }^{3}$, \\ H. Schechtman', \\ M. Pelajo-M achado ${ }^{1}$, \\ B.S. Vale ${ }^{1}$, M.S. Panasco ${ }^{1}$ \\ and J.A. Lenzi ${ }^{1}$
}

\author{
1Departamento de Patologia, Instituto O swaldo Cruz, and \\ 2Programa de Computação Científica, Presidência, \\ Fundação 0 swaldo Cruz, Rio de Janeiro, RJ, Brasil \\ ${ }^{3}$ Department of Agricultural Engineering, Technion, \\ Israel Institute of Technology, Haifa, Israel
}

\section{Correspondence \\ H.L. Lenzi \\ Departamento de Patologia \\ Instituto O swaldo Cruz, FIOCRUZ \\ Avenida Brasil, 4365 \\ 21045-900 Rio de Janeiro, RJ \\ Brasil \\ Fax: +55-21-598-4350 or 598-4466, \\ or 590-3495 \\ E-mail: hlenzi@gene.dbbm.fiocruz.br}

Presented at the 5th Brazilian

Symposium on Extracellular

Matrix - SIMEC, Angra dos Reis,

RJ, Brasil, September 7-10, 1998.

Research supported by FIOCRUZ and CN Pq. M. Pelajo-M achado is supported by CAPES and M.S.

Panasco is supported by FAPERJ.

\section{Abstract}

The collagen structure of isolated and in situ liver granuloma from Swiss Webster mice infected with Schistosoma mansoni was sequentially and three-dimensionally analyzed during different times of infection (early acute, acute, transitional acute-chronic, and chronic phases) by laser scanning confocal microscopy and electron scanning variable vacuum microscopy. The initial granuloma structure is characterized by vascular collagen residues and by anchorage points (or fiber radiation centers), from where collagenous fibers are angularly shed and self-assembled. During the exudative-productive stage, the self-assembly of these fibers minimizes energy and mass through continuous tension and focal compression. The curvature or angles between collagen fibers probably depends on the fibroblastic or myofibroblastic organization of stress fibers. Gradually, the loose unstable lattice of the exudative-productive stage transforms into a highly packed and stable architecture as a result of progressive compactness. The three-dimensional architecture of granulomas provides increased tissue integrity, efficient distribution of soluble compounds and a haptotactic background to the cells.

A granuloma is characterized by the presence of clusters of cells of the "mononuclear phagocyte series" with or without other cell types (1). The clusters of mononuclear cells are compact (organized) (2), and epithelioid cells, in some cases, define the typical granulomatous lesion (3). Granulomas function as little organs, presenting stages of maturation and involution during their development, and final disappearance. Granulomas arise from
Key words

- Granuloma

- Schistosoma mansoni

- Collagen

- Extracellular matrix

- Confocal microscopy colonial aggregations of monocyte/macrophages, sometimes forming giant syncytial cells. The development of a granuloma depends on the etiologic agent, which may be intracellular (e.g. mycobacteria), intra- and extracellular (e.g. Paracoccidioides brasiliensis) or extracellular (4). Schistosomal granulomas are the best example of the latter category and are the subject of the present study. 
Figure 1 - (All the figures, except 7 and 8, represent black and white images of liver granulomas originated from 3-D reconstructions made by CSLM). Exudative-productive stage with three (internal, medial or paracentral and external) defined layers. The external layer is expanded and forms a loose stroma favoring perigranulomatous hemopoietic metaplasia. The medial layer shows a honeycomb arrangement of collagen fibers, seen also in Figure 7. The central layer is devoid of collagen in the periovular subregion, and presents a smaller amount of fibers, which are thin.

Figures 2 and 3 - Initial exudative-productive stage presenting a mesh-like pattern of collagen fibers, showing several isolated fiber anchorage points (fiber radiation centers) (arrows).

Figure 4 - Productive stage with a compact concentric medial layer.
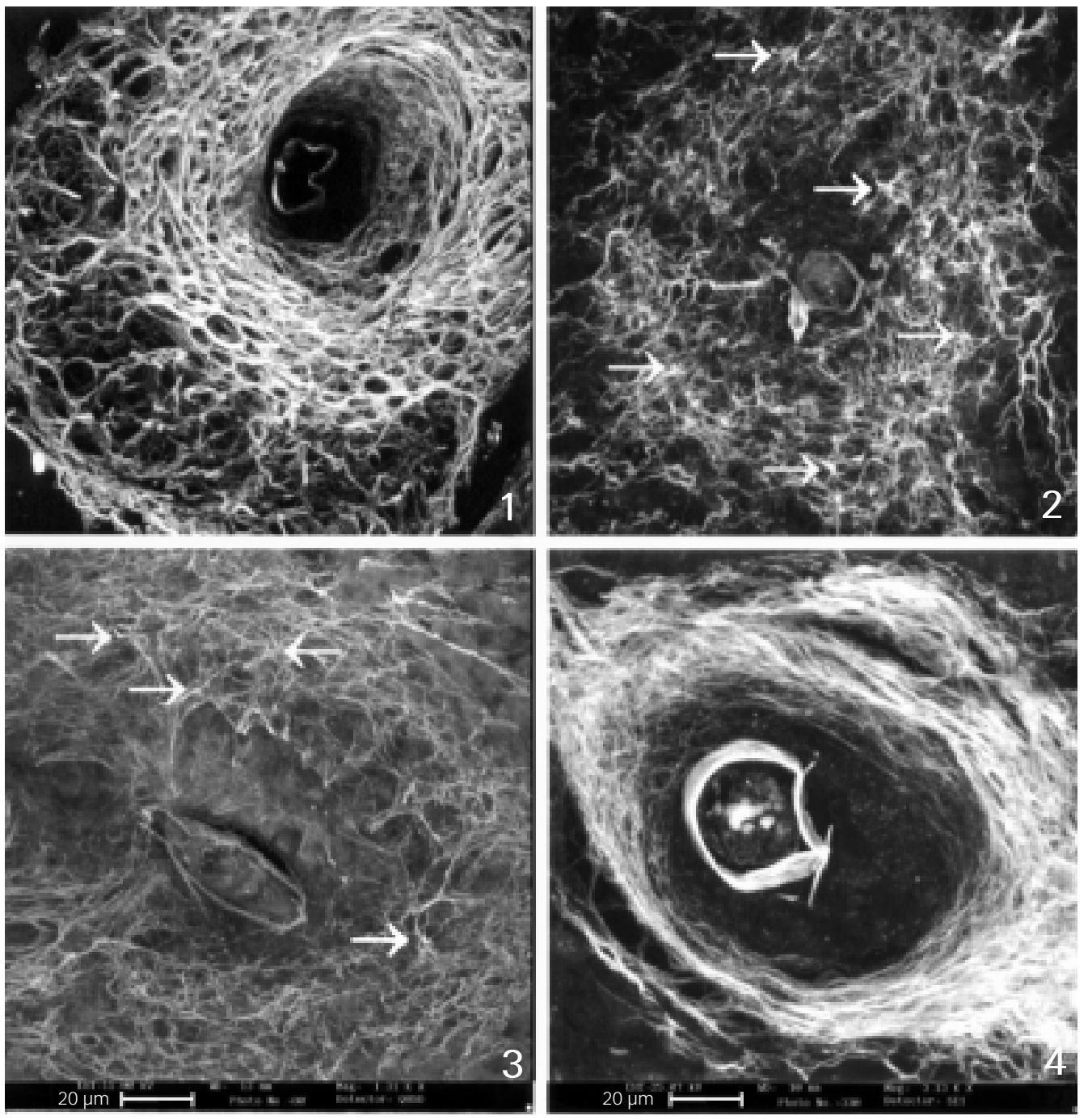

Granuloma size in acute Schistosoma mansoni infection is determined primarily by $\mathrm{T}$ cell reactivity, and is, therefore, sensitive to IL-10 $(5,6)$, while the antibody has a minor effect (7). In contrast, in chronic infections, downregulation of granuloma size requires an antibody and downmodulated $\mathrm{T}$ cells (8). The regulation of fibrosis in the liver may differ from the regulation of granuloma size (7,9-11).

The histoarchitecture of hepatic schistosomal granulomas was examined by threedimensional analysis of isolated or in situ granulomas from Swiss Webster mice. The animals were infected by percutaneous exposure to 70 cercariae of the Belo Horizonte
(BH) isolate of $S$. mansoni. The mice were killed at the following times after infection: $35,40,45,60,70,90,120$ and 180 days. Liver fragments were homogenized for 2 min in a blender and the suspensions were filtered through grids to select isolated granulomas of two different sizes: smaller and larger than $300 \mu \mathrm{m}$ (12). Purified granulomas and liver fragments were fixed in Carson's modified Millonig's phosphatebuffered formalin, $\mathrm{pH} 7.4$ (13), and embedded in paraffin. Thick histological sections were stained with phosphomolybdic acidpicrosirius red (PMA-PSR) (14) for threedimensional study of collagenous fibers by confocal laser scanning microscopy (CLSM) 

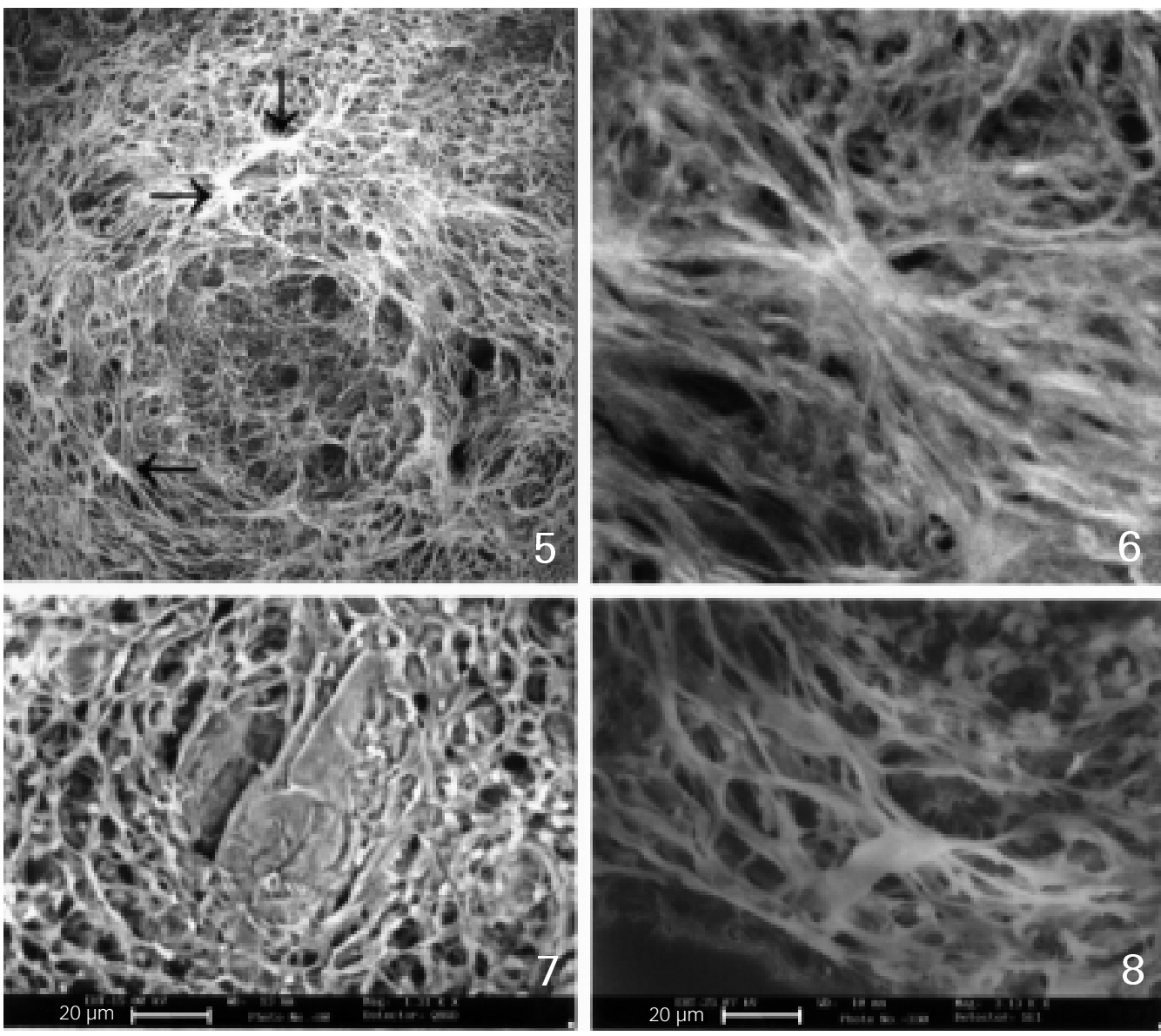

(LSM 410, Zeiss, Jena, Germany). Deparaffined sections were desiccated in an incubator at $37^{\circ} \mathrm{C}$ for $1 \mathrm{~h}$, and analyzed by scanning electron microscopy (SEM), low-vacuum mode (435-VP, Leo, Cambridge, England).

We observed, as previously described (15), that the granuloma stage in the liver is preceded by a lytic pre-granulomatous phase (exudative phase) which produces a spherical space by destruction of the parenchyma for the final establishment and organization of the lesion. In the granulomatous stage, cellular adhesion and sorting play an important role. The effects of aggregation and sorting create different zones in the mature granuloma structure, where the combination of different types of cells consistently forms an internal layer (peri-ovular), enveloped by paracentral and external layers (Figure 1). Probably the cells with stronger mutual ad- hesion aggregate in the center, whereas less cohesive cells remain on the surface. Cells appear to migrate from the less adhesive (external layer) to the more cohesive layer (internal layer rich in CD44+ cells) (data not shown) through an adhesive haptotactic gradient provoked by egg products. They follow a centripetal migration in a diffusiongenerated radial concentration gradient ("direct migration" model) (16).

The three-dimensional tissue architecture of the granuloma, as observed by CLSM and SEM (variable vacuum), presents extracellular matrix (ECM) fibers which are initially arranged in a mesh pattern (Figures 2 and 3), evolving to a final and compact concentric arrangement (Figure 4). These two patterns of fiber arrangement correspond to two different collagen populations which appear successively during granuloma evolution:
Figure 5 - Exudative-productive stage with intense fiber compactness, clearly showing several fiber anchorage points (fiber radiation centers) (arrows).

Figures 6 and 8 - Details of fiber anchorage points or fiber radiation centers seen by CSLM (Figure 6) and by TEM (low vacuum) (Figure 8). From the anchorage points the fibers are emitted or projected under restricted angles.

Figure 7 - Honeycomb aspect of the medial layer (TEM - low vacuum). 
collagen type III containing reticular fibers is later replaced by collagen type I containing collagen fibers (17).

The arrangement and distribution of the collagen network, even in its initial mesh pattern, seems to be preferentially oriented toward the internal secretory cells (fibroblasts and/or myofibroblasts), aiming to hold in place the tissues surrounding the new organoid structure.

The curvature or angles between collagen fibers probably depends on the fibroblast or myofibroblast organization. In fact, these cells form anchorage points, i.e. fiber radiation centers, from where collagen fibers are shed and self-assembled (Figures 5, 6 and 8) sometimes forming a honeycomb arrangement (Figures 1 and 7).

The large amount of ECM produced by granuloma cells appears to be independent of the surrounding forces, and thus emphasizes the biological role, compared to the mechanical role, of ECM molecules in the formation of the granuloma. This is also supported by the observation that independently of the organ (e.g. liver, lung, intestine, pancreas, milky spot) or site (superficial (subcapsular or subpleural) or deep in the center of the parenchyma) where the granulomas are located, they always tend to be spherical. Moreover, when granulomas were mechanically cut, there was no extrusion of their content from the core to the periphery (data not shown), which indicates that their structure does not present internal pressure counterbalanced by tension in the fibrous envelope (external layer).
The structure, which is formed by rearrangement into concentric layers, seems to be based upon tension and/or adhesion-dependent forces. If so, due to a process of minimization of energy, the granuloma would develop in a spherical form, as this is the form in which opposing forces pull equally from all directions and are in balance (15).

The loose unstable lattice of the exudative-productive stage metamorphoses into a highly packed, stable architecture as a result of progressive compactness. Probably, both matrix-specific and shape (or stress)-specific effects also activate different genes involved in the granuloma maturation and involution.

In conclusion, a schistosomal granuloma is not a tangled web of individual cells and fibers, but an organized structure, where the three-dimensional arrangement of its fibers originating from anchorage points (or fiber radiation centers) serves to provide increased tissue integrity, efficient distribution of soluble compounds (growth factors and cytokines) and a haptotactic background to the cells. Thus, in granuloma development there is a synergistic process in that the function of the fully organized structure ("little macrophagic metazoa") is literally greater than the sum of the actions of its individual cells and ECM fibers or components.

\section{Acknowledgments}

The authors wish to thank Ms. Luzia de Fátima Gonçalves Caputo and Adelaide Lopes Amorim for technical assistance.

\section{References}

1. Turk J L (1992). Granulomatous disease. In: McGee J OD, Isaacson PG \& Wright NA (Editors), Oxford Textbook of Pathology. Oxford University Press, Oxford, 394406.

2. Adams DO (1983). The biology of the granuloma. In: Ioachim HL (Editor), Pathology of Granulomas. Raven Press, New York, 1-20.
3. Mariano M (1995). The experimental granuloma. A hypothesis to explain the persistence of the lesion. Revista do Instituto de Medicina Tropical de São Paulo, 37: 1-15.

4. Lenzi HL, Lenzi J A, Kerr IB, Antunes SLG, Mota EM \& Oliveira DN (1991). Extracellular matrix in parasitic and infectious diseases. Memórias do Instituto Oswaldo
Cruz, 86 (Suppl III): 77-90.

5. Flores-Villanueva PO, Zheng XX, Strom TB \& Stadecker MJ (1996). Recombinant IL-10 and IL-10/ Fc treatment down-regulates egg antigen-specific delayed hypersensitivity reactions and egg granuloma formation in schistosomiasis. J ournal of Immunology, 156: 3315-3320.

6. Wynn TA, Morawetz R, Scharton-Kersten 
T, Hieny S, Morse III HC, Kuhn R, Muller W, Cheever AW \& Sher A (1997). Analysis of granuloma formation in double cytokine deficient mice reveals a central role for IL-10 in polarizing both th1 and th2type cytokine responses in vivo. J ournal of Immunology, 159: 5014-5023.

7. Olds GR, Meneza S el, Mahmoud AAF \& Kresina TF (1989). Differential immunoregulation of granulomatous inflammation, portal hypertension and hepatic fibrosis in murine schistosomiasis mansoni. J ournal of Immunology, 142: 36053611.

8. J ankovic D, Cheever AW, Kullberg MC, Wynn TA, Yap G, Caspar P, Lewis FA, Clynes R, Ravetch J V \& Sher A (1998). CD4+ cell-mediated granulomatous pathology in schistosomiasis is downregulated by a B cell-dependent mechanism requiring Fc receptor signaling. J ournal of Experimental Medicine, 187: 619-629.

9. Phillips $S M$, Ramadan $M A$, Zekavat $A M$, Hilliard B, Sugaya H el \& Rafaei M (1996). Regulation of the schistosome granuloma and fibrosis by TGFß-1 and antifibrotic chemoterapy-safronil. American J ournal of Tropical Medicine and Hygiene, 55: 191 (Abstract).

10. Cheever AW (1997). Differential regulation of granuloma size and hepatic fibrosis in schistosome infections. Memórias do Instituto Oswaldo Cruz, 92: 689-692.

11. Cheever AW, Jankovic D, Yap GS, Kullberg MC, Sher A \& Wynn TA (1998). Role of cytokines in the formation and downregulation of hepatic circumoval granulomas and hepatic fibrosis in Schistosoma mansoni-infected mice. Memórias do Instituto Oswaldo Cruz, 93: 25-32.

12. Cotta-Pereira G, Lenzi HL, Oliveira DN, Wajsenzoni IJ R \& Lenzi J A (1991). On the presence of elastic microfibrils in liver granuloma of murine schistosomiasis mansoni. Memórias do Instituto Oswaldo Cruz, 86 (Suppl III): 129-130.

13. Carson FL, Martin J H \& Lynn J A (1973). Formalin fixation for electron microscopy: A re-evaluation. American J ournal of Clinical Pathology, 59: 365-373.
14. Dolber PC \& Spach MS (1993). Conventional and confocal fluorescence microscopy of collagen fibers in the heart. J ournal of Histochemistry and Cytochemistry, 41: 465-469.

15. Lenzi $H L$, Kimmel $E$, Schechtman $H$, Pelajo-Machado M, Romanha WS Pacheco RG, Mariano $M$ \& Lenzi J A (1998). Histoarchitecture of schistosomal granuloma development and involution: Morphogenetic and biomechanical approaches. Memórias do Instituto Oswaldo Cruz, 93 (Suppl I): 141-151.

16. Steinberg MS (1996). Adhesion in development: Historical overview. Developmental Biology, 180: 377-388.

17. J unqueira LCU, Montes GS, Toledo OMS \& J oazeiro PP (1986). Morphological, histochemical and biochemical observations on the connective tissue matrix of in situ and isolated hepatic granulomas in experimental murine schistosomiasis. Annals of Tropical Medicine and Parasitology, 80: 27-41. 